\title{
Correlative Microscopy of Cerebellar Molecular Layer Postsynaptic Proteins and Postsynaptic Receptors
}

\author{
Orlando J Castejón ${ }^{1 *}$ and Haydee Viloria de Castejón ${ }^{2}$ \\ ${ }^{1}$ Department of Biological Research Institute, Venezuela \\ ${ }^{2}$ Department of Medicine, Venezuela
}

*Corresponding author: Orlando J Castejón, Department of Biological Research Institute, Instituto de Investigaciones Biológicas, Venezuela.

To Cite This Article: Orlando J Castejón, Haydee Viloria de C. Correlative Microscopy of Cerebellar Molecular Layer Postsynaptic Proteins and Postsynaptic Receptors. Am J Biomed Sci \& Res. 2019 - 1(2). AJBSR.MS.ID.000514. DOI: 10.34297/AJBSR.2019.01.000514

Received: January 12, 2019 | Published: January 23, 2019

\begin{abstract}
Correlative microscopy of cerebellar molecular layer allowed us to image the localization of postsynaptic receptors using a comparative and intermicroscopy study applying transmission electron microscopy (TEM), freeze-etching and direct replicas for transmission electron microscopy (FFTEM), field emission scanning electron microscopy (FESEM), and confocal laser scanning microscopy (CLSM) using immunohistochemistry of Synapsin-I and PSD-95, immunohistochemistry of $\mathrm{Ca}^{2+} /$ calmodulin dependent protein kinase II alpha and immunohistochemistry GluR1 subunits of AMPA receptors alpha. These techniques showed the images of postsynaptic densities of parallel fibers-Purkinje dendritic ramifications containing postsynaptic proteins and glutamate postsynaptic receptors. The freeze-etching replica method for TEM showed the three-dimen $\leftarrow$ sional structure and intramembrane morphology of postsynaptic ellipsoidal and spheroid intramembrane particles. Confocal laser scanning microscopy using Synapsin-I (Syn-I) and PSD-95, GluR1 subunits of ionotropic glutamate receptors, and $\mathrm{Ca}^{2+} /$ calmodulin dependent protein kinase II alpha contribute to image the precise localization of postsynaptic receptors. The high resolution FESEM showed 25-50nm globular subunits at the spine postsynaptic density corresponding to the localization of postsynaptic proteins and/or postsynaptic glutamate receptors.
\end{abstract}

Keywords: Electron microscopy; Confocal microscopy; Postsynaptic receptors

\section{Introduction}

Purkinje dendritic spines have been studied for more than one century since Ramon and Cajal pioneering studies with the light microscope. The advent of electron microscopy and the freeze etching technique made possible to visualize spine morphology and its intramembrane morphology [1-6]. The development of confocal laser scanning microscopy and the immunohistochemistry techniques [7] allowed us to study the domains of mayor proteins inside the postsynaptic region of Purkinje dendritic spines [8-10] The postsynaptic density (PSD) is a dynamic multi-protein complex attached to the postsynaptic membrane composed of several hundred proteins such as receptors and channels, scaffolding and adaptor proteins, cell-adhesion proteins, cytoskeletal proteins, G-proteins and their modulators and signaling molecules including kinases and phosphatases [11-13]. Glutamate receptors are the most abundant excitatory neurotransmitter receptors in the brain, responsible for mediating the vast majority of excitatory transmission in neuronal networks. The AMPA- and NMDAtype ionotropic glutamate receptors (iGluRs) are ligand-gated ion channels that mediate the fast-synaptic responses, while metabotropic glutamate receptors (mGluRs) are coupled to downstream signaling cascades that act on much slower timescales [14-15]. Tao et al. [16] have developed an integrative approach combining cryo-electron tomography and correlative fluorescence microscopy to visualize 3D ultrastructural features of intact excitatory and inhibitory synapses in their native state. Yun-Hong et al. [17], by using the immunoabsorption method, reported binding partner proteins of two proteins residing in the interiors, PSD-95 and $\alpha$-tubulin, and those of two proteins residing in the peripheral regions, elongation factor- $1 \alpha$ and calcium, calmodulin-dependent protein kinase II $\alpha$ subunit, of cerebral and cerebellar postsynaptic densities (PSDs). Nanoscopy studies, ranging from quantification of receptors and scaffolding proteins in postsynaptic densities and 
their dynamic behavior, to imaging of synaptic vesicle proteins and dendritic spines in living neurons or even live animals has been carried out by means of super resolution microscopy [18].

In the present paper we used a combined intermicroscopy study of postsynaptic Purkinje dendritic spines by means of transmission electron microscopy and freeze etching technique, field emission scanning microscopy and confocal laser scanning microscopy immunohistochemistry methods in an attempt to get insight into the molecular composition of postsynaptic density and image postsynaptic receptors.

\section{Material and Methods}

\section{Transmission electron microscopy (TEM)}

For transmission electron microscopy (TEM), slices 1-2mm thick of mouse cerebellar cortex were immediately fixed by immersion in $4 \%$ glutaraldehyde in $0.1 \mathrm{M}$ phosphate buffer solution (pH 7.4) for $4-16 \mathrm{~h}$ at $4^{\circ} \mathrm{C}$; they were post fixed for $1 \mathrm{~h}$ in a similarly buffered $1 \%$ osmium tetroxide solution, dehydrated through graded concentrations of ethanol and embedded in Araldite. Thin sections were stained with uranyl and lead salts and observed with a JEOL 100B elec $\leftarrow$ tron microscope.

\section{Freeze-etching and direct replicas for transmission electron microscopy (FFTEM) [3]}

The freeze-etching direct replica technique was applied to the cerebellar cortex of adult Swiss albino mice. The brains were carefully removed, and thin $1-2 \mathrm{~mm}$ slices of the cerebellar cortex were fixed in $1 \%$ ice-cold glutaraldehyde $0.1 \mathrm{M}$ phos $\leftarrow$ phate buffer, $\mathrm{pH}$ 7.2-7.4, for $1 \mathrm{~h}$. All cut pieces were immersed in three changes of $25 \%$ glycerol in a similar buffer for $30 \mathrm{~min}$, mounted on gold discs and frozen in Freon at N2 temperature for 3-5sec. They were immediately trans-ferred to a Balzer BAF-301 freeze-fracture unit equipped with an electron beam gun, at $-110^{\circ} \mathrm{C}$, in a vacuum of 4 X 106 or better. Fractured surfaces were shadowed with a layer of carbon-platinum about $2.5 \mathrm{~nm}$ thick. Replicas were floated off on water, cleaned in Chlorox overnight, rinsed in water, bathed in $50 \%$ $\mathrm{H} 2 \mathrm{SC}>4$ and rinsed in multiple changes of water. Cleaned replicas were mounted on grids usually coated with parlodion or Formvar films and examined with a JEOL 100B electron microscope at $80 \mathrm{kV}$.

\section{Field emission scanning electron microscopy (FESEM) [4]}

Albino mouse cerebellar cortex was excised, cut into 1-2 mm slices and immersed in $4 \%$ glutaraldehyde in $0.1 \mathrm{M}$ phos $\leftarrow$ phate buffer solution, $\mathrm{pH} 7.4$, for $24 \mathrm{~h}$ at $4{ }^{\circ} \mathrm{C}$; and post-fixed for $1 \mathrm{~h}$ in a similarly buffered $1 \%$ osmium tetroxide solution.

\section{Delicate specimen preparation}

Specimens were dehydrated in a graded series of ethanol (30, $50,70,80,902 \times 100 \%$ ) prior to wrapping individual tissue pieces in preformed absolute ethanol filled parafilm cryofrac-ture packets. Rapid freezing of packets was performed by plunging into LN2.
First, the packet was transferred from the LN2 storage vessel with LN2 chilled forceps in order to avoid thermal damage. Secondly, the cooled fracture blade was removed from the LN2, the packet was orientated under the blade, and immediately struck with a heavy tool. Fractured tissue fragments were transferred into chilled absolute etlianol $\left(4^{\circ} \mathrm{C}\right)$ and thawed. Tissues were loaded into fresh absolute ethanol filled mesh baskets within the boat of a Polaron E-3000 critical point drier. The boat was then loaded into the Tissues were loaded into fresh absolute ethanol filled mesh baskets within the boat of a Polaron E-3000 critical point drier. The boat was then loaded into the drier, and exchanged with $\mathrm{CO}_{2}$ gas at a rate of $1.21 / \mathrm{min}$. The CPD chamber was then thermally regulated to the critical temperature and decompressed at the same rate. Dried speci-mens (shiny face up), were mounted onto aluminium stubs $9 \mathrm{mmX} 2 \mathrm{mmX} 1 \mathrm{~mm}$ for the ISI DS-130 SEM with silver paste and degassed at $2 \mathrm{X} 107$ torr prior to sputter coating.

\section{Metal coating for FESEM imaging}

Specimens were chromium coated with a continuous $1 \mathrm{~nm}$ film in a Denton DV-602 turbo pumped sputter deposition system operated in a vacuum of Argon at 5X103 torr.

\section{High resolution field emission scanning electron microscopy [19]}

Specimens were staged in-lens of an ISI DS-130F equipped with Schottky field emitter. The instrument was operated at accelerating voltages of $5 \mathrm{kV}$ in order to produce a small spot size and minimal specimen penetration while attaining excellent adequate signal to noise ratio at all magnifications. Images were digitally recorded in $116 \mathrm{sec}$ with TIF-files size of 4.8 megabytes, contrast processed with Adobe Photoshop.

\section{Synapsin-I and PSD-95 immunohistochemistry [7]}

Animals were used in accordance with NIH and institutional guidelines. For synapsin-I and PSD-95 immunohistochemistry, cerebellar cortex slices were derived from 14-21 days old rats. After decapitation, the lateral cerebellar lobules were removed from posterior fossa and placed in ice-cold $\left(4^{\circ} \mathrm{C}\right)$ Hanks balanced salt solution supplemented with dextrose $(6 \mathrm{mg} / \mathrm{ml})$. Fragments of cerebellar cortex were transversally sliced $(300 \mu \mathrm{m}$ thick sections) using a manual tissue chopper (Stoelting). The slices were immediately fixed in $4 \%$ paraformaldehyde in ice-cold 0.1M phosphate-buffered saline (PBS). All samples for PSD-95 immunolabeling were chemically fixed in ice cold paraformaldehyde for no more than $15 \mathrm{~min}$ to preserve antigenicity. Free floating slices were placed in a multi-well plate for subsequent rinsing, blocking and labeling steps.

Slices were rinsed in PBS ( $3 \times 5 \mathrm{~min})$ and then extracted overnight in 1\% Triton X-100 in PBS (Polysciences). Blocking was done with $50 \mathrm{mM} \mathrm{NH} 4 \mathrm{Cl}$ in PBS (30min), followed by $20 \%$ horse serum in PBS (30min). Washing (5min) was done with $1 \%$ horse serum in PBS (HSPBS). All subsequent steps were carried out in 
1\% HSPBS. Slices were double labeled with anti-synapsin-I rabbit IgG polyclonal antibody (diluted 1:300; Molecular Probes, Eugene, OR) and anti-PSD-95 (mouse) diluted 1:200 (Alexis, San Diego, CA). These primary antibodies were applied overnight $(4 \stackrel{\circ}{\circ} \mathrm{C})$, and then tissues were rinsed again ( $3 \times 15 \mathrm{~min}$ ) in HSPBS. The secondary antibodies used were Alexa-488 goat anti-rabbit IgG and Alexa-568 goat-anti-mouse IgG (GAM; Molecular Probes), diluted 1: 500, and applied overnight $\left(4^{\circ} \mathrm{C}\right)$. Tissues were then rinsed $3 \times 15 \mathrm{~min}$ in $1 \%$ HSPBS, mounted on microscope slides, sealed with vacuum grease in a closed chamber containing 1\% HSPBS, and covered with a coverslip. To visualize Purkinje cell bodies and dendrites, samples were immunolabeled with rabbit anti-calbindin antibodies (1:5001:2000; Chemicon). Some tissue slices were triple-labeled with antibodies against PSD-95, Synapsin-I, and Calbindin. Primary and secondary antibodies against PSD-95 and Synapsin-I were applied first, then primary antibodies against calbindin were applied followed by Cy5 as a secondary antibody.

\section{Immunohistochemistry of $\mathrm{Ca}^{2+}$ /calmodulin dependent protein kinase II alpha by means of confocal laser scanning microscopy [7]}

Animals were used in accordance with NIH and institutional guidelines. For CaMKII alpha labeling, cerebellar cortex slices were derived from 14 -days-old postnatal (P14) rats anesthetized with $\mathrm{CO}_{2}$. After decapitation, the lateral lobules of cerebellum were removed from posterior fossa and placed in ice-cold $\left(4^{\circ} \mathrm{C}\right)$ Hanks balanced salt solution (HBSS) supplemented with dextrose $(6 \mathrm{mg} / \mathrm{ml})$. Fragments of cerebellar cortex were transversally sliced $(300 \mu \mathrm{m}$ thick sections) using a manual tissue chopper (Stoelting). The slices were immediately fixed in $4 \%$ paraformaldehyde (PF) in ice-cold $0.1 \mathrm{M}$ phosphate-buffered saline (PBS) for 48 hours. Free-floating slices were placed in a multiwell plate for subsequent rinsing, blocking and labeling steps. Slices were rinsed in PBS (2 x 5 minutes) and then extracted overnight in 1\% Triton X-100 in PBS (Polysciences). Blocking was done with $50 \mathrm{mM} \mathrm{NH4Cl}$ in PBS (30 $\mathrm{min}$ ), followed by $20 \%$ horse serum in PBS (30min). Washing was done with $1 \%$ horse serum in PBS (HSPBS) (5 min). All subsequent steps were carried out in 1\% HSPBS. Slices were double labeled with a primary antibody against CaMKII alpha, and a secondary antibody the Alexa 488 goat anti mouse (GAM)-antibody (diluted 1:300; Molecular Probes, Eugene, OR). The labeling process was applied overnight $\left(4^{\circ} \mathrm{C}\right)$, and tissues were then rinsed $3 \times 15 \mathrm{~min}$ in $1 \%$ HSPBS, mounted on microscope slides, sealed with vacuum grease in a closed chamber containing 1\% HSPBS, and covered with a coverslip.

\section{Immunohistochemistry GluR1 subunits of AMPA receptors alpha by means of confocal laser scanning microscopy [7]}

Animals were used in accordance with NIH and institutional guidelines. For GluR1 immunohistochemistry, cerebellar cortex slices were derived from 14-day-old postnatal (P14) rats anesthetized with $\mathrm{CO}_{2}$. After decapitation, the lateral lobules of cerebellum were removed from posterior fossa and placed in icecold $\left(4{ }^{\circ} \mathrm{C}\right)$ Hanks balanced salt solution (HBSS) supplemented with dextrose $(6 \mathrm{mg} / \mathrm{ml})$. Fragments of cerebellar cortex were transversally sliced $(300 \mu \mathrm{m}$ thick sections) using a manual tissue chopper (Stoelting). The slices were immediately fixed in $4 \%$ paraformaldehyde (PF) in ice-cold 0.1M phosphate-buffered saline (PBS). All samples for GluR1 immunolabeling were chemically fixed in ice cold PF for no more than $30 \mathrm{~min}$ to preserve antigenicity. Free-floating slices were placed in a multiwell plate for subsequent rinsing, blocking and labeling steps. Slices were rinsed in PBS $(2 \mathrm{x}$ 5 minutes) and then extracted overnight in 1\% Triton X-100 in PBS (Polysciences). Blocking was done with $50 \mathrm{mM} \mathrm{NH4Cl}$ in PBS (30 $\mathrm{min}$ ), followed by $20 \%$ horse serum in PBS (30 min). Washing was done with $1 \%$ horse serum in PBS (HSPBS) (5 min). All subsequent steps were carried out in 1\% HSPBS. Slices were double labeled with anti-GluR1 monoclonal antibody (diluted 1:500; Molecular Probes, Eugene, OR). This primary antibody was applied overnight (4oC), and then tissues were rinsed again ( $3 \times 15$ minutes) in HSPBS. The secondary antibody used was Alexa-488 goat anti-rabbit (GAR) IgG (Molecular Probes), diluted 1:300, and applied overnight (4oC). Tissues were then rinsed $3 \times 15$ min in $1 \%$ HSPBS, mounted on microscope slides, sealed with vacuum grease in a closed chamber containing 1\% HSPBS, and covered with a coverslip.

\section{Result}

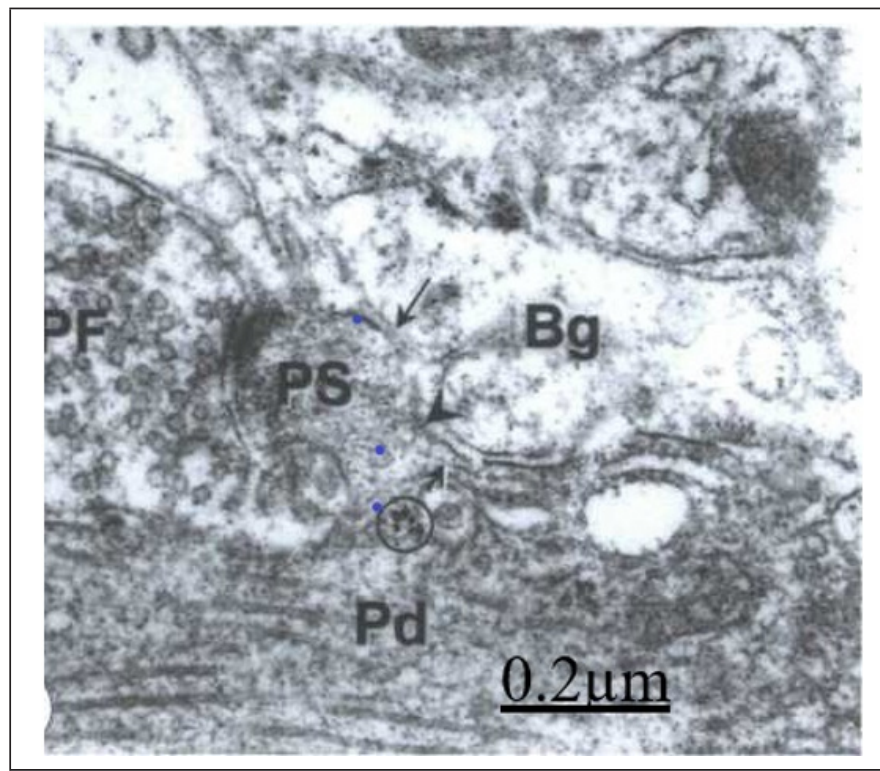

Figure 1: TEM images of ultrathin sections of a Purkinje dendrite (PD) showing a mushroom-shaped spines (arrow) making synaptic connection with a presynaptic ending of a parallel fiber (PF). The Bergmann glial cell cytoplasm $(\mathrm{Bg})$ surround the spine synaptic contact. The arrowhead points out the dark extracellular space between the Bergmann glial cell and the dendritic spine. Ribosomes are visualized at the base of spine neck (circle).

Transmission electron microscopy (TEM) shows the Purkinje tertiary dendritic ramifications emitting mushroom-shaped dendritic spines and making synaptic connection with the 
presynaptic ending of parallel fibers at the outer third cerebellar molecular layer (Figure 1).

\section{The freeze-etching replica method for TEM}

The freeze-etching replica method for TEM showed the three- dimen $\leftarrow$ sional structure and intramembrane morphology of Purkinje dendritic ramification and their spines. About 90 IMPs were observed in the spine head, and about 65 IMPs at the short neck (Figure 2).

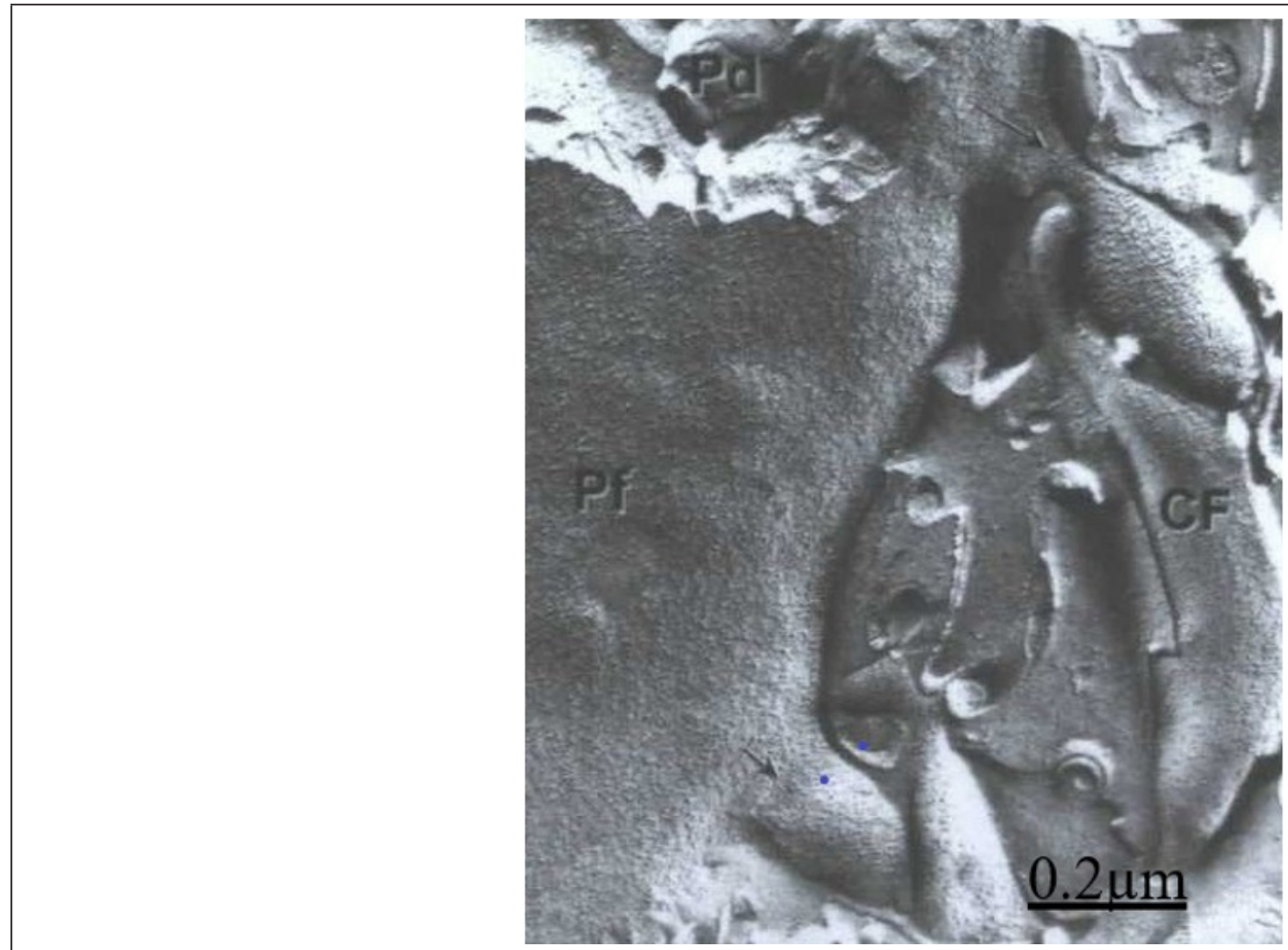

Figure 2: Transmission electron microscopy and freeze etching technique. Purkinje cell (Pd) dendritic membrane showing the intramembrane particle distribution (PF) of dendritic shaft and two spines (arrows), A climbing fiber (CF) is seen making an axospino dendritic contact.

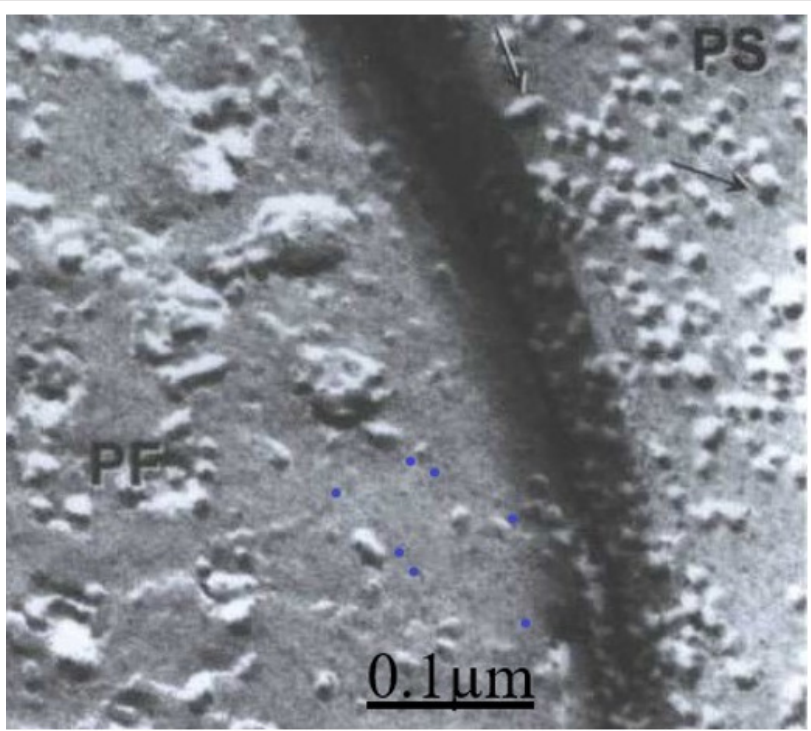

Figure 3: Mouse cerebellar cortex. Parallel fiber (PF)-Purkinje spine (PS)synapse showing the PF face of synaptic vesicles at the presynaptic ending and the small medium and large ellipsoidal and spheroid intramembrane particles (arrow) at the postsynaptic membrane.

Transmission electron microscopy and freeze etching technique showed a high magnification the presynaptic vesicles at the presynaptic ending of parallel fiber ending and the ellipsoidal or spheroid intra-memmbrane particles at the postsynaptic membrane of Purkinje dendritic spine (Figure 3). 
Synapsin-I (Syn-I) and PSD-95 immunohistochemistry of rat cerebellar cortex Dailey

The comparative localization of two prominent synaptic proteins, Synapsin-I (Syn-I) and PSD-95, was observed in slices of developing postnatal day rat cerebellar cortex using double fluorescence immunohistochemistry and multi-channel laser scanning confocal microscopy. The pre- and postsynaptic endings of parallel fiber-Purkinje spine synapses and the basket cell axonal endings upon Purkinje cell bodies were specifically immunohistochemistry imaged (Figure 4).

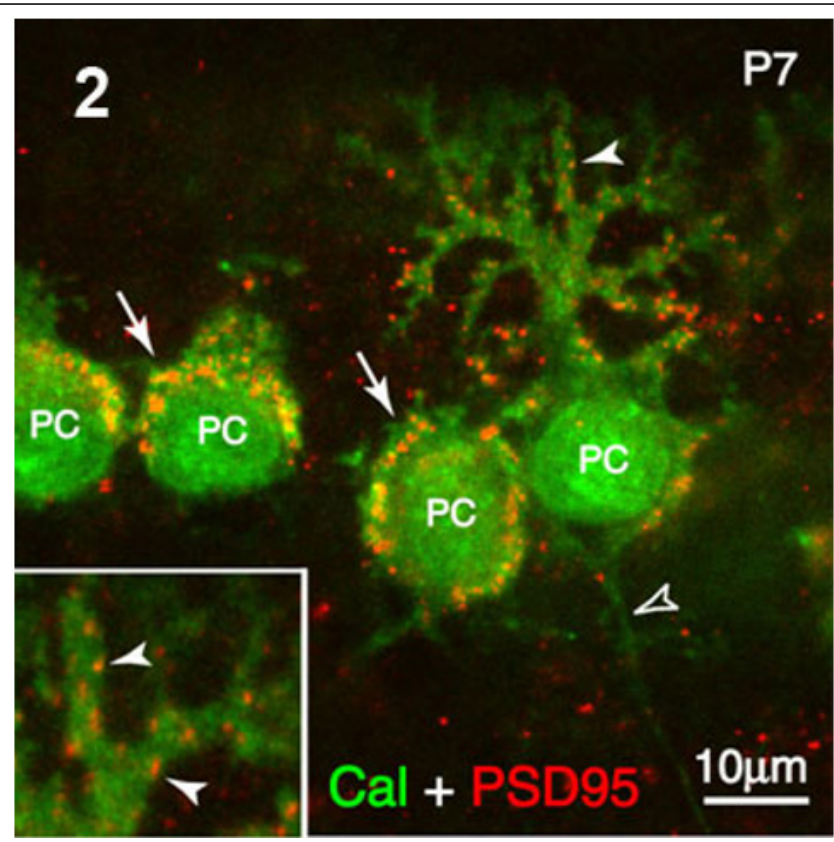

Figure 4: Rat cerebellar cortex. Immunohistochemistry of Calbinding and PSD95 immunostaining showing the postsynaptic sites of axosomatic synapses on Purkinje cell (PC) bodies (arrows) and the axodendritic synapses at the level of Purkinje dendritic arborization (arrowheads). These latter have been illustrated at the left lower level square.

\section{Confocal laser scanning Immunohistochemistry of} GluR1 subunit of rat cerebellar cortex

Immunohistochemistry of GluR1 subunits of ionotropic glutamate receptors of rat cerebellar cortex exhibited a strong and small puntacte staining at the level of inner, middle, and outer thirds molecular layer surrounding Purkinje cell secondary and tertiary dendritic branches, corresponding to the localization of climbing and parallel fiber-Purkinje spine synapses. These immunostainings showed the localization of GluR1 subunits of ionotropic glutamate receptors in the inhibitory Purkinje neurons of cerebellar cortex and its association with excitatory glutamatergic cerebellar circuits. GluR1 strong immunoreactivity was confined to Purkinje cell body and Purkinje cell dendritic arborization at the molecular layer (Figure 5).

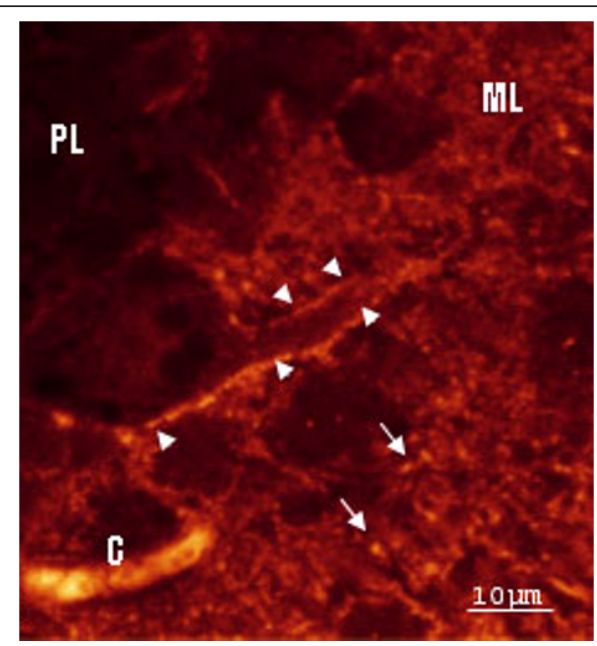

Figure 5: Rat cerebellar cortex. Immunohistochemistry of GluR1 subunit- A strong and small puntacte staining also was found at the inner, middle, and outer thirds molecular layer surrounding Purkinje cell secondary and tertiary dendritic branches, corresponding to the localization of climbing and parallel fiber-Purkinje spine synapses. 
The immunohistochemistry of $\mathrm{Ca}^{2+} /$ calmodulin dependent protein kinase II alpha (CaMKII)

The immunohistochemistry of $\mathrm{Ca}^{2+} /$ calmodulin dependent protein kinase II alpha (CaMKII) in rat developing cerebellar cortex depicted the immunopositively reaction as small puncta at the outer third molecular layer corresponding to the localization of parallel fiber-Purkinje spine synapses (Figure 6).

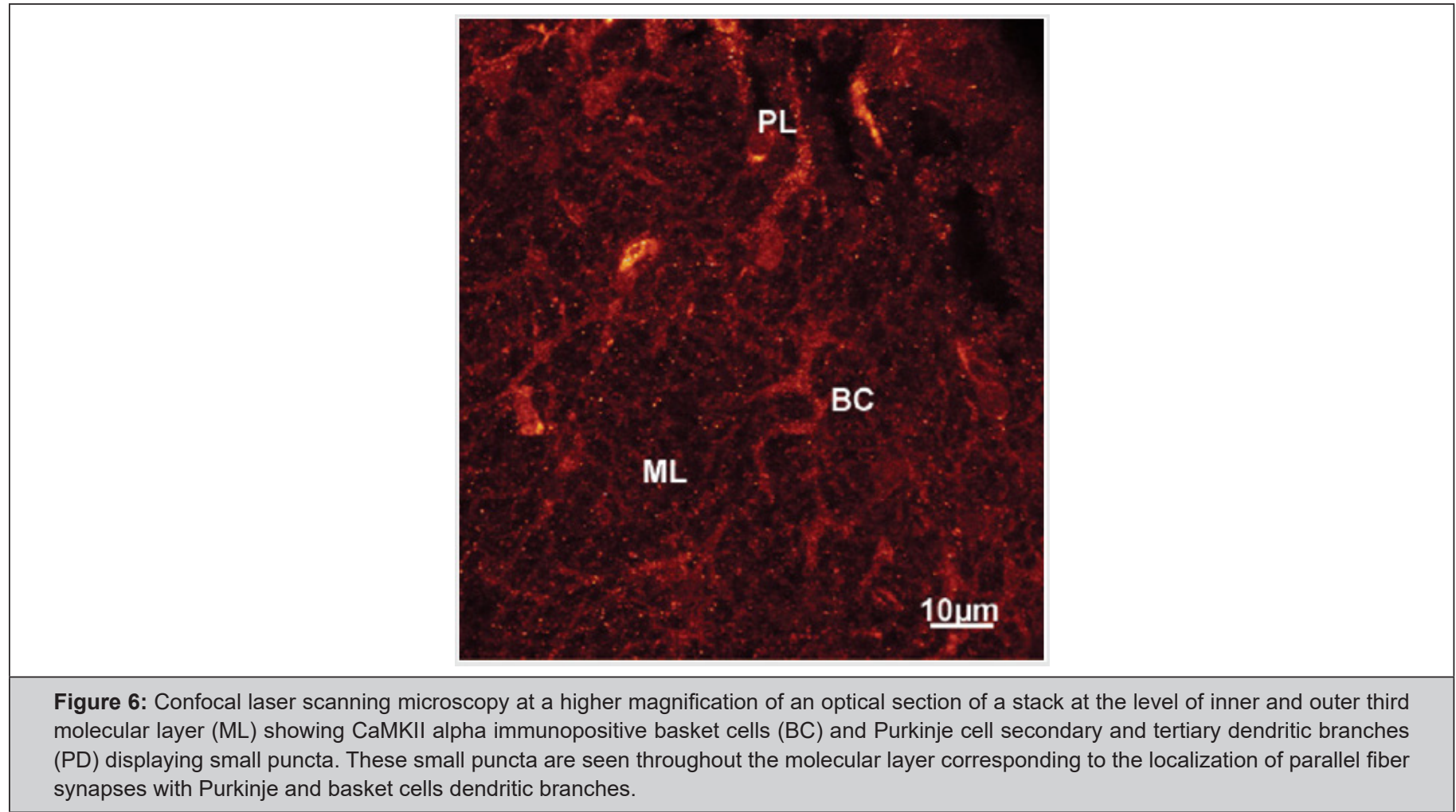

Low resolution FESEM of mouse cerebellar cortex elongated and lanceolate Pds separated by 100-500 nm on the molecular layer dendritic shaft surface (Figure 7), The criofracture process has

Low resolution FESEM showed unattached mushroom-type, displayed the dendritic spine body and necks (Figure 7).

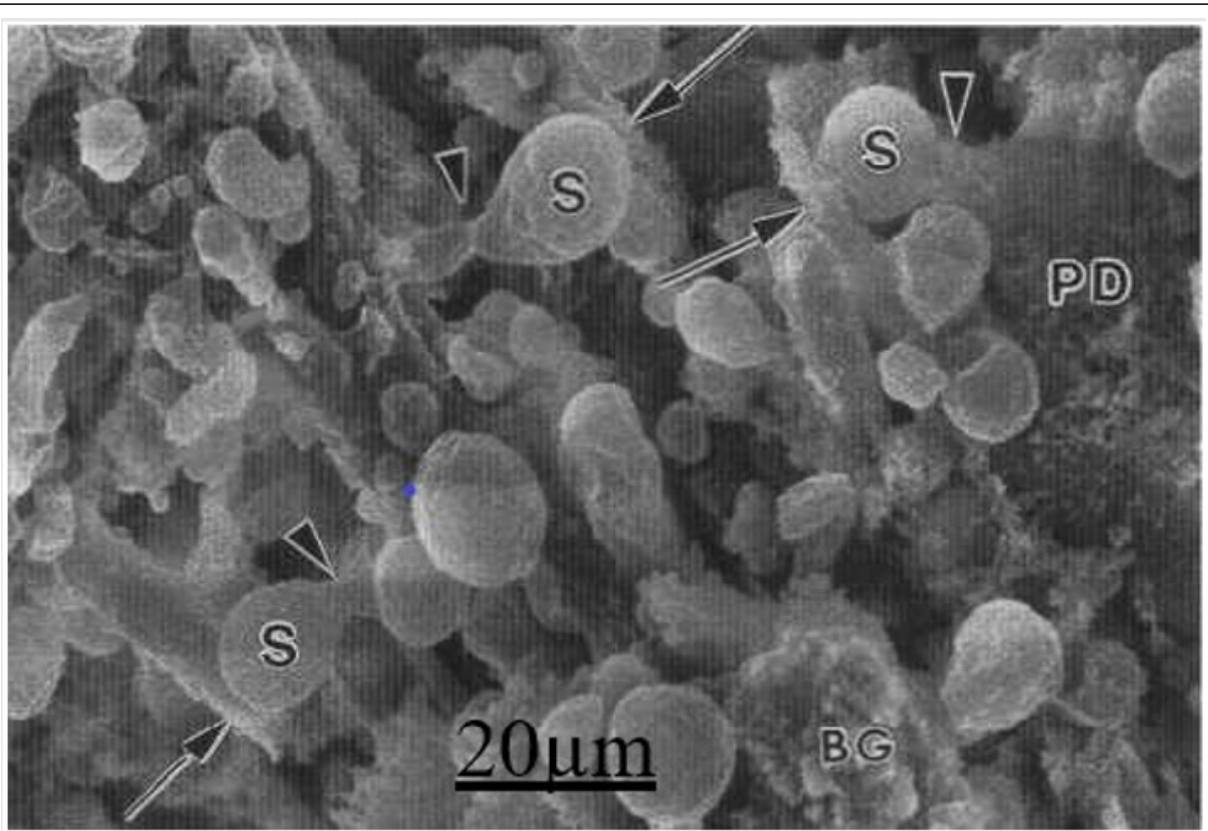

Figure 7: Low resolution FESEM showing the Purkinje (PD) dendritic spines (S) and their attached necks (arrowheads) to the tertiary Purkinje dendrite (arrows). The remaining Bergmann glial cell cytoplasm (BG) also is seen. 


\section{High resolution field emission scanning electron microscopy}

The field emission scanning electron microscopy showed the fractured presynaptic endings of cerebellar parallel fibers or granule cell axons in the cerebellar molecular layer containing the synaptic vesicles aggregated toward the presynaptic membrane, the homogenous dense presynaptic axoplasm and the SEI profile of nonspecialized presynaptic membrane (Figure 8).

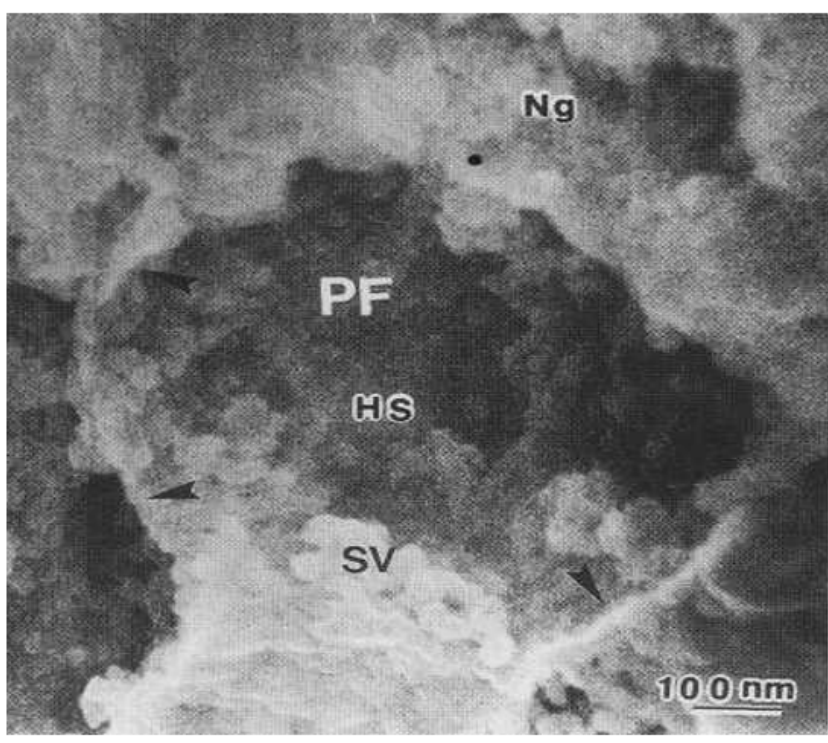

Figure 8: High magnification FE SEM of a parallel fiber presynaptic ending showing the SE-I imaging of a fractured presynaptic ending of cerebellar parallel fiber (PF) showing clustered synaptic vesicles (SV) attached to the presynaptic membrane. The presynaptic homogenous substance (HS), and the non-specialized sites presynaptic membrane (arrowheads) also are distinguished.

High resolution FESEM showed the presynaptic dense projection, the synaptic vesicles, the pre- and postsynaptic membranes, the synaptic cleft and the 25-50 nm globular subunits at the spine postsynaptic density corresponding to the localization of postsynaptic proteins and/or postsynaptic receptors (Figure 9).

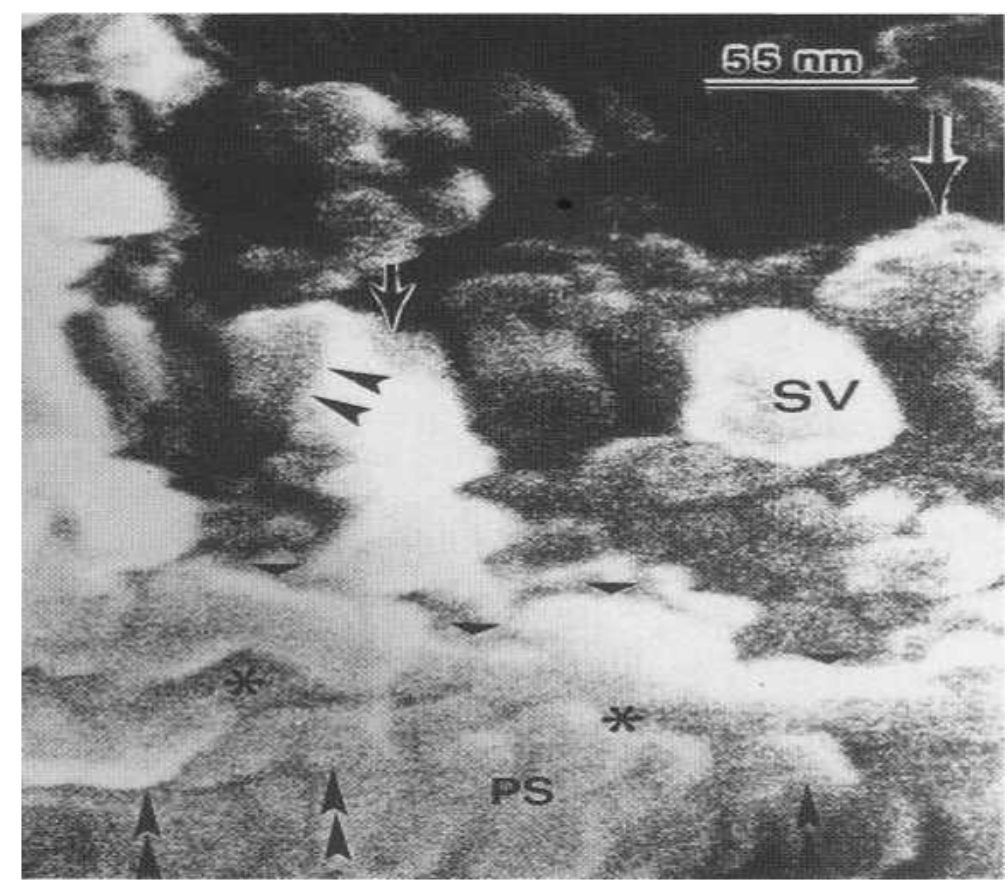

Figure 9: High FESEM showing the synaptic vesicle (SV), the presynaptic dense projection (arrowheads), the presynaptic membrane (triangles), the synaptic cleft (asterisks) and the postsynaptic membrane with attached globular subunits (double head arrows) within the postsynaptic density (PS).

The aggregated globular subunits can be observed at the postsynaptic ending as round substructures 8 (Figure 10). 


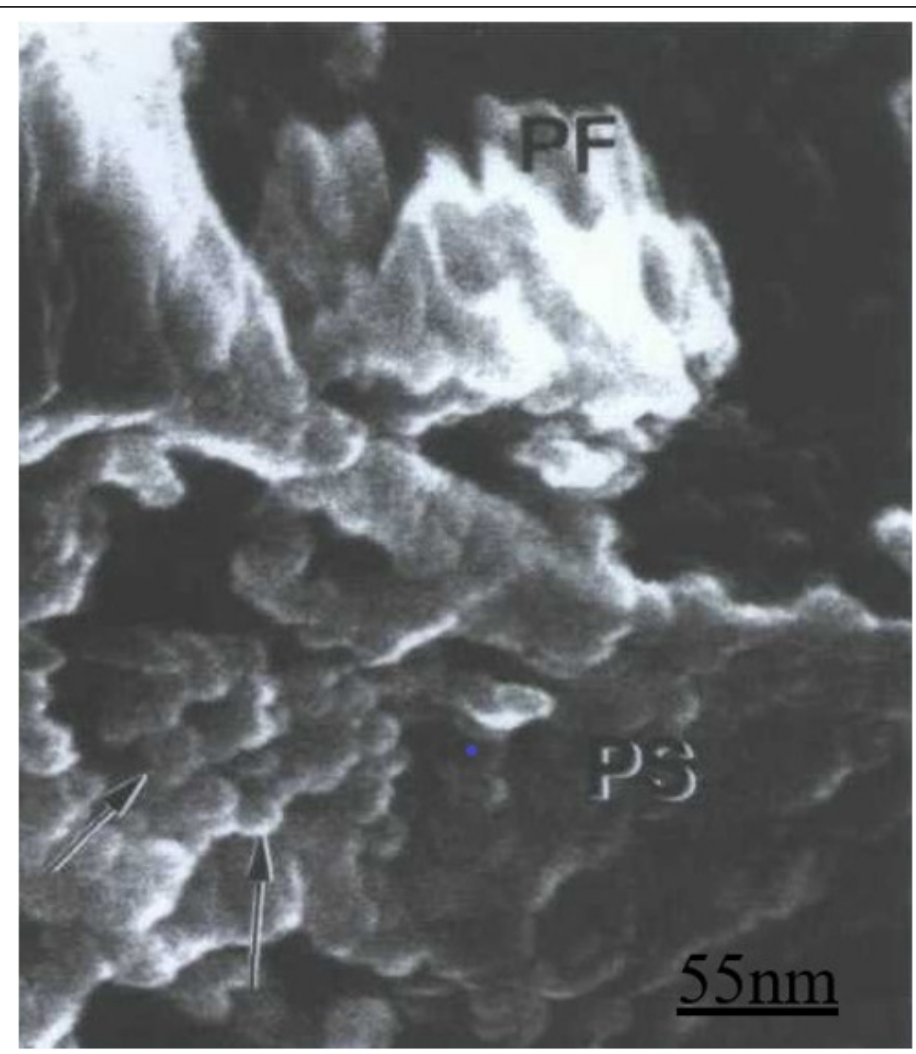

Figure 10: Higher magnification of FESEM showing the presynaptic dense projection of parallel fiber (PF) presynaptic ending and the postsynaptic density (PS) containing the clustered globular subunits corresponding to the postsynaptic receptors (arrows).

\section{Discussion}

\section{Transmission electron microscopy and freeze-etching replica technique}

In earlier investigations we studied a freeze-fracture scanning electron microscopy and comparative freeze-etching study of parallel fiber-Purkinje spine synapses of vertebrate cerebellar cortex (Castejon,1990). Lately we reported a combined conventional and high resolu $\leftarrow$ tion field emission scanning electron microscopy of vertebrate cerebel $\leftarrow$ lar parallel fiber-Purkinje spine synapses [4-6, 20-22]. These previous studies demonstrated the potentiality and resolution power of correlative and inter microscopy studies and their related technique to approach to the study of synaptic receptors.

The hypothesis that some proteins of the postsynaptic membrane are locally synthesized at postsynaptic sites [23] is supported by our finding of aggregates of ribosomes found at the site of emergency of postsynaptic spines. We have used the TEM and freeze etching technique to study protein membrane morphology of postsynaptic spines and intramembrane particle distribution. Recently, there has been a major revival in freeze-fracture electron microscopy thanks to the development of effective ways to reveal integral membrane proteins by immunogold labeling. One of these methods is known as detergent-solubilized Freeze-fracture Replica Immunolabeling (FRIL). The distinctive appearance of the postsynaptic membrane specialization of glutamatergic synapses further allows, upon labeling of ionotropic glutamate receptors, to quantify and analyze the intrasynaptic distribution of these receptors [24]. Immunogold labeling shows that the presynaptic active zone provides a scaffold for key molecules involved in the release of neurotransmitter, whereas the postsynaptic density contains ligand-gated ionic channels, other receptors, and a complex network of signaling molecules [13]. Freeze etching replicas exposed postsynaptic intramembrane particles that can be correlated with the globular subunits observed at high resolution FESEM.

\section{Synapsin-I and PSD-95, GluR1 and CaMKII alpha immunohistochemistry}

In the present study we have found positive immunoreaction for Synapsin-I and PSD-95, GluR1 and CaMKII. The GluR1 protein, a 106KDA glycoprotein, appears predominantly in synaptic plasma membranes, where it is enriched in the postsynaptic density [25]. The GluR1 forms $\mathrm{Ca}^{2+}$ permeable channels and exhibit inward rectification [26,27]. CaMKII alpha presence in the main excitatory and inhibitory circuits of developing cerebellar cortex is presumably related with its participation in information, motor learning and memory processes. Our findings support the role of CaMKII as a molecular switch that is capable of storing long-term synaptic memory as postulated by Lismann et al. [28]. According to Hansel et al. [29] alpha CaMKII is essential for motor learning 
and long term potentiation on hippocampus, cerebral cortex and Purkinje cells of cerebellum. PSD 95, a main member of MAGUK family, interacts directly with carboxyl termini of NMDA receptor subunits and clusters them to the postsynaptic membrane. In addition, PSD 95 is involved in binding and organizing proteins connected with NMDAR signaling [12].

\section{High resolution field emission scanning electron microscopy}

In an intent to extract relevant information from the outer and inner surfaces of pre- and postsynaptic endings, we have applied chromium coating to mouse cerebellar cortex. This continuous method film, $1 \mathrm{~nm}$ thick, markedly eliminated the decoration artifacts introduced by gold-palladium coating. Accurate topographic contrast is generated by FESEM by the SE-I and SEII electrons specifically emanating from the specimen surface. Scanning electron micrographs in which the SE-I are accentuated relative to SE-II are obtained when sam $\leftarrow$ ples are coated with a thin continuous film (1-2nm) of chromium and examined in an 'in lens' SEM, designed to eliminate the SE-III signal as carried out in the present study. In chromium coated nerve tissue we have generated a defined contrast mechanism in which the topography of the sample is precisely delineated [4].

Hundreds of molecules have been identified in the PSD: iongated and G-protein-coupled receptors, association, adaptors, and scaffolding proteins, key enzymes involved in phosphorylationdephosphorylation mechanisms, and cytoskeletal proteins. Each of these proteins may have a pivotal function in setting the molecular scenario for the development of synaptic plasticity. Scaffolding proteins are major players in the organization of the postsynaptic signal transduction machinery, they regulate receptor trafficking and clustering, modulate axon path finding, and drive the correct targeting of neuronal proteins to their appropriate cytoplasmic compartment. Emerging findings suggest a relevant involvement of PSD scaffolding/adaptor proteins in behavior modulation in animal models of synaptic plasticity disorders and pharmacological isomorphisms [30]. PSD scaffolds proteins such as members of the MAGUK (membrane-associated guanylyl kinase), Shank (SH3 domain and ankyrin repeat-containing protein) and Homer families interact simultaneously with different kinds of receptors and modulate their function by linking the receptors to downstream signaling events. For example, PSD 95, a main member of MAGUK family, interacts directly with carboxyl termini of NMDA receptor subunits and clusters them to the postsynaptic membrane. In addition, PSD 95 is involved in binding and organizing proteins connected with NMDAR signaling. Based on the modular character and ability to form multiproteins interactions, MAGUK, Shank and Homer are perfectly suited to act as a major scaffold in postsynaptic density [12].

Previous studies by means of biochemical methods, electron microscopy and confocal laser scanning microscopy have localized the CaMKII at the level of postsynaptic density, being central to the regulation of glutamates synapses. According to Lisman et al. [28] previous work indicates that a binding pattern for CaMKII is the NMDA receptor within the postsynaptic density. The AMPA receptor subunit GluR1 is also phosphorylated by CaMKII enhancing channel function. Significant differences were found in protein composition and organization across PSDs from the different brain regions. The signaling protein, $\beta$ CaMKII, was found to be a major component of each PSD type and was more abundant than $\alpha$ CaMKII in both hippocampal and cerebellar PSDs. The scaffold molecule PSD95, a major component of cortical PSDs, was found absent in a fraction of cerebellar PSDs and when present was clustered in its distribution. In contrast, immunogold labeling for the proteasome was significantly more abundant in cerebellar and hippocampal PSDs than cortical PSDs. Together, these results indicate that PSDs exhibit remarkable diversity in their composition and morphology, presumably as a reflection of the unique functional demands placed on different synapses Significant differences were found in protein composition and organization across PSDs from the different brain regions. The signaling protein, $\beta$ CaMKII, was found to be a major component of each PSD type and was more abundant than $\alpha$ CaMKII in both hippocampal and cerebellar PSDs [31].

To address the critical aspect of synaptic organizational dynamics, Li \& Blanpied [32] performed single-molecule tracking of transmembrane proteins using universal point accumulationfor-imaging-in-nanoscale-topography (UPAINT) over PSDs whose internal structure was simultaneously resolved using photoactivated localization microscopy (PALM). Their results provide important experimental confirmation that PSD scaffold protein density strongly influences the mobility of transmembrane proteins.

\section{Futures avenues for research on postsynaptic receptors}

Because numerous proteins thought to be involved in establishing PSD structure are linked to disorders including autism and depression, this motivates further exploration of how PSD nanostructure is created. The combined application PALM and uPAINT should be invaluable for distinguishing the interactions of mobile proteins with their nano-environment both in synapses and other cellular compartments [30]. More recently considerable advances in super-resolution microscopy have begun deciphering the sub diffraction organization of synaptic elements and their functional roles. In particular, the dynamic nanoscale organization of neurotransmitter receptors in the postsynaptic membrane has recently been suggested to play a major role in various aspects of synapstic function. The super-resolution imaging techniques have overcome the classical barrier imposed by the diffraction of light and can now resolve the localization and dynamic behavior of proteins within small compartments with nanometer precision, revolutionizing the study of dendritic spine architecture [14,3335]. Biomedical applications of pathology of postsynaptic receptors 
should be promising lines of research on the study of hyperactivity and attention deficit, autism spectrum disorder epilepsy, mood disorders, schizophrenia, and degenerative diseases, such as Parkinson and Alzheimer diseases.

\section{Conclusion}

Transmission electron microscopy and freeze etching replica technique revealed the presence of intramembrane morphology of postsynaptic density proteins. Confocal laser scanning microscopy and immunohistochemistry of GluR1subunits displayed showed the localization of GluR1 subunits of ionotropic glutamate receptors in the inhibitory Purkinje neurons of cerebellar cortex and its association with excitatory glutamatergic cerebellar circuits. The confocal laser scanning microscopy and immunoimmunohistochemistry of $\mathrm{Ca} 2+/$ calmodulin dependent protein kinase II alpha (CaMKII) in rat developing cerebellar cortex depicted small puncta at the cerebellar outer third molecular layer corresponding to the localization of parallel fiber-Purkinje dendritic spine synapses. FEHRSEM imaged the globular subunits at the postsynaptic density corresponding to the glutamate receptors. The localization of these globular subunits corresponds to the postsynaptic IMPs visualized with the freeze-fracture replica methods. This correlative microscopy study offers new views on the participation of these postsynaptic receptors on information processing in the cerebellar cortex.

\section{Acknowledgement}

This work has been partially carried out through a subvention obtained from National Institute of Health. (NIH). Grant NS 17150 to Michael Dailey and CONDES LUZ subvention to Orlando Castejon. We deeply appreciate the collaboration of Dr. Robert Apkarian at the Microscope Unit of Yerkes Research Center, Emory University (1992-1993) by the use of high resolution field emission SEM.

\section{References}

1. Gray EG (1961) The granule cells, mossy synapses and Purkinje spine synapses of the cerebellum: Light and electron microscope observations. J Anat 95: 345-356.

2. Palay SL, Chan-Palay V (1974) Cerebellar Cortex, Cytology and Organization. Berlin: Springer-Verlag.

3. Castejón OJ (1990) Freeze-fracture scanning electron microscopy and comparative freeze-etching study of parallel fiber-Purkinje spine synapses of vertebrate cerebellar cortex. J Submicrosc Cytol Pathol 22: 281-295.

4. Castejón OJ, Apkarian RP (1992) Conventional and high-resolution scanning electron microscopy of outer and inner surface features of cerebellar nerve cells. J Submicrosc Cytol Pathol 24: 549-562.

5. Castejón OJ, Castejón HV, Apkarian RP (1994) High resolution scanning electron microscopy features of primate cerebellar cortex. Cell Mol Biol 40: 1173-1181.

6. Castejon OJ Castejón HV (1997) Conventional and high resolution scanning electron microscopy of cerebellar Purkinje cells. Biocell 21(2): 149-160.
7. Dailey ME (2002) Optical imaging of neural structure and physiology: Confocal fluorescence microscopy in live brain slices. In: 'Brain Mapping: The Methods'. 2nd ed., Elsevier Science USA, pp 49-76.

8. Castejón OJ, Castellano A, Arismendi G, Apkarian R (2004) Correlative microscopy of Purkinje dendritic spines: a field emission scanning and transmission electron microscopic study. J Submicrosc Cytol Pathol 36(1): 29-36.

9. Castejón OJ, Fuller L, Dailey ME (2004) Localization of synapsin-I and PSD-95 in developing postnatal rat cerebellar cortex. Develop Brain Res 151: $25-32$.

10. Castejón OJ, Dailey ME (2009) Immunohistochemistry of GluR1 subunits of AMPA receptors of rat cerebellar nerve cells. Biocell 33: 71-80.

11. Bergersen L, Waerhaug O, Helm J, Thomas M, Laake P, et al. (2001). A novel postsynaptic density protein: the monocarboxylate transporter MCT2 is co-localized with delta-glutamate receptors in postsynaptic densities of parallel fiber-Purkinje cell synapses. Exp Brain Res 136(4): 523-534.

12. Beresewicz M (2007) Scaffold proteins (MAGUK, Shank and Homer in postsynaptic density in the central nervous system. Postepy Biochem 53(2): 188-197.

13. Harris KM, Weinberg RJ (2012) Ultrastructure of synapses in the mammalian brain. Cold Spring. Harb Perspect Biol 4(5): a005587.

14. Scheefhals N, MacGillavry HD (2018) Functional organization of postsynaptic glutamate receptors. Mol Cell Neurosci 91:82-94.

15. Twomey EC, Sobolevsky AI (2018) Structural mechanisms of gating in ionotropic glutamate receptors. Biochemistry 57(3):267-276.

16. Tao CL, Liu YT, Sun R, Zhang B, Qi L (2018) Differentiation and characterization of excitatory and inhibitory synapses by cryo-electron tomography and correlative microscopy. J Neurosci 38(6): 1493-1510.

17. Yun-Hong Y, Chih-Fan C, Chia-Wei C, Yen-Chung C (2011) A study of the spatial protein organization of the postsynaptic density isolated from porcine cerebral cortex and cerebellum. Mol Cell Proteomics 10(10): M110.007138.

18. Willig KI, Barrantes FJ (2014) Recent applications of super resolution microscopy in neurobiology. Curr Opin Chem Biol 20:16-21.

19. Castejón OJ, Apkarian RP (1993) Conventional and high resolu-tion field emission scanning electron microscopy of vertebrate cerebel-lar parallel fiber-Purkinje spine synapses. Cell Mol Biol (Noisy Le Grand) 39: 863-873.

20. Castejón OJ (1993) Sample preparation techniques for conventional and high resolution scanning electron microscopy of the central nervous system. The cerebellum as a model Scanning Microsc 7: 725-740.

21. Castejon OJ (1996) Conventional and high resolution scanning electron microscopy of cerebellar synaptic junction. Scanning Microsc 10: 177186.

22. Castejón OJ, Apkarian RP, Castejón HV, Alvarado MV (2001) Field emission scanning electron microscopy and freeze-fracture transmission electron microscopy of mouse cerebellar synaptic contacts. J Submicrosc Cytol Pathol 33(3): 289-300.

23. Rao A, Steward O (1991) Evidence that protein constituents of postsynaptic membrane specializations are locally synthesized: analysis of proteins synthesized within synaptosomes. J Neurosci 11(9):28812895.

24. Schönherr S, Seewald A, Kasugai Y, Bosch D, Ehrlich I (2016) F combined optogenetic and freeze-fracture replica immunolabeling to examine input-specific arrangement of glutamate receptors in the mouse amygdala. J Vis Exp (110).

25. Blackstone CD, Moss SJ, Martin LJ, Levey AI, Price DL, et al. (1992) Biochemical characterization and localization of a non-N-methyl-Daspartate glutamate receptor in rat brain. J Neurochem 58: 1118-1126. 
26. Pellegrini-Giampietro DE, Bennett MV, Zukin RS (1992) Are $\mathrm{Ca}^{(2+)}$ permeable kainate/AMPA receptors more abundant in immature brain? Neurosci Lett 144: 65-69.

27. Petralia RS, Wang YX, Zhao HM, Wenthold RJ (1996) Ionotropic and metabotropic glutamate receptors show unique postsynaptic presynaptic, and glial localizations in the dorsal cochlear nucleus. J Comp Neurol 372(3): 356-383.

28. Lisman J, Schulman H, Cline H (2002) The molecular basis of CaMKI function in synaptic and behavioural memory. Nat Rev Neurosci 3(3):175-190.

29. Hansel C, de Jeu M, Belmeguenai A, Houtman SH, Buitendijk GH, et al. (2006) AlphaCaMKII is essential for cerebellar LTD and motor learning. Neuron 51(6): 835-843.

30. de Bartolomeis A, Fiore G (2004) Postsynaptic density scaffolding proteins at excitatory synapse and disorders of synaptic plasticity: implications for human behavior pathologies. Int Rev Neurobiol 59: 221-254.
31. Farley MM, Swulius MT, Waxham MN (2015) Electron tomographic structure and protein composition of isolated rat cerebellar, hippocampal and cortical postsynaptic densities. Neuroscience 304:286-301.

32. Li TP, Blanpied TA (2016) Control of transmembrane protein diffusion within the postsynaptic density assessed by simultaneous singlemolecule tracking and localization microscopy. Front Synaptic Neurosci 8:19.

33. Compans B, Choquet D, Hosy E (2016) Review on the role of AMPA receptor nano-organization and dynamic in the properties of synaptic transmission. Neurophotonics 3(4): 041811.

34. Cajal SR (1911) Histologie du Système Nerveux de l'Homme et des Vertebres. Consejo Superior de Investigaciones Científicas (Editors) Instituto Ramón y Cajal Madrid España.

35. Castejón OJ (1968) Observaciones microscópico-electrónicas a nivel de la capa molecular de la corteza cerebelosa. Tesis Doctoral Facultad de Medicina Invest Clín 27: 57-108. 